\title{
ON THE HEWITT REALCOMPACTIFICATION OF A PRODUCT SPACE
}

\author{
BY \\ W. W. COMFORT( ${ }^{(1)}$
}

One of the themes of Edwin Hewitt's fundamental and stimulating work [16] is that the $Q$-spaces (now called realcompact spaces) introduced there, although they are not in general compact, enjoy many attributes similar to those possessed by compact spaces; and that the canonical realcompactification $v X$ associated with a given completely regular Hausdorff space $X$ bears much the same relation to the ring $C(X)$ of real-valued continuous functions on $X$ as does the Stone-Čech compactification $\beta X$ to the ring $C^{*}(X)$ of bounded elements of $C(X)$. Much of the Gillman-Jerison textbook [12] may be considered to be an amplification of this point. Over and over again the reader is treated to a " $\beta$ " theorem and then, some pages later, to its " $v$ " analogue.

One of the most elegant " $\beta$ " theorems whose " $v$ " analogue remains unproved and even unstated is the following, given by Irving Glicksberg in [13] and reproved later by another method in [11] by Zdeněk Frolík: For infinite spaces $X$ and $Y$, the relation $\beta(X \times Y)=\beta X \times \beta Y$ holds if and only if $X \times Y$ is pseudocompact. The present paper is an outgrowth of the author's unsuccessful attempt to characterize those pairs of spaces $(X, Y)$ for which $v(X \times Y)=v X \times v Y$. It is shown (Theorem 2.4) that, barring the existence of measurable cardinals, the relation holds whenever $Y$ is a $k$-space and $v X$ is locally compact; and more generally (Theorem 4.5 ) that the relation holds whenever the $k$-space $Y$ and the locally compact space $v X$ admit no compact subsets of measurable cardinal. The question arises naturally as to when it will occur that $v X$ is locally compact. Our best result in this direction, a part of Theorem 4.8, is, again, not definitive: $X$ is locally pseudocompact if and only if there is a locally compact space $Y$ for which $X \subset Y \subset v X$.

Some of the questions treated here are susceptible to attack when thrown into the uniform space context. See Onuchic [18] and, more intensively, Isbell [17, especially Chapter 8].

I am indebted to Tony Hager for several references, and for allowing me to announce here that, in addition to the results of [15], he has achieved certain new theorems on the relation $v(X \times Y)=v X \times v Y$, which being "uniform" and not "topological" have negligible overlap with those of this paper.

Presented to the Society, January 27, 1967, under the title Locally compact realcompactifications; received by the editors June 6, 1966 and, in revised form, December 19, 1966.

(1) The author gratefully acknowledges support from the National Science Foundation under contract NSF GP-5750. He also thanks the Research Council of the University of Massachusetts for support received from Faculty Research Grant FR-J37-67(1). 
My chief debt is to Stelios Negrepontis, with whom [7] was coauthored. I consider the first half of the present paper to be a collection of corollaries to Theorem 2.8 of [7].

I am indebted to the referee for the very elegant construction which appears in $\S 5$, answering fully a question posed in [5] and in an early version of this paper. His kindness in supplying the validating arguments in considerable detail is much appreciated, as is his willingness (indeed, his generous insistence) that the example appear in this paper, under my name. The referee has improved this paper further by shortening one of the proofs, and by requesting the excision of certain "overripe literary flourishes" which marred the original version. For all this, we thank the referee.

1. Preliminaries. Every space hypothesized or constructed in this paper is a completely regular Hausdorff space. The ring of real-valued continuous functions on such a space $X$ is denoted $C(X)$, and its subring of bounded functions $C^{*}(X)$. If $A \subset X$ and each element of $C(A)$ is the restriction to $A$ of an element of $C(X)$, then we say (as in [12]) that $A$ is $C$-embedded in $X$; the expression " $A$ is $C$ *embedded in $X$ "' is defined analogously.

Each space $X$ is simultaneously dense and $C^{*}$-embedded in exactly one compact space, denoted $\beta X$ and called the Stone-Čech compactification of $X$. Analogously, each space $X$ is simultaneously dense and $C$-embedded in exactly one realcompact space, denoted $v X$ and called the Hewitt realcompactification of $X$. Proofs and extensive illuminating discussions appear in [12].

A space $X$ is called pseudocompact if it admits no unbounded real-valued continuous function-i.e., if $C(X)=C^{*}(X)$. A nonpseudocompact space is always $C^{*}$-embedded, but never $C$-embedded, in $\beta X$, and there are many less trivial examples which illustrate that the two concepts ( $C$-embedded, $C^{*}$-embedded) differ. The following result, therefore, quoted from [7], is unexpected.

1.1 Theorem. If $X \times Y$ is $C^{*}$-embedded in $v X \times v Y$, then $X \times Y$ is $C$-embedded in $v X \times v Y$. In this case $v(X \times Y)=v X \times v Y$.

The Glicksberg-Frolík theorem quoted earlier contains no cardinality restrictions on the spaces involved. The casual reader is not likely to be impressed by the good fortune attendant to this fact until he tries in earnest to discover its realcompact analogue. The following two results, which are perhaps the major results of [7], show that no discussion of the relation $v(X \times Y)=v X \times v Y$ can be complete without a consideration of the possible existence of measurable cardinals (defined below).

1.2 THEOREM. If $Y$ is a compact space of nonmeasurable cardinal, then $v(X \times Y)=$ $v X \times Y=v X \times v Y$ for each space $X$.

1.3 EXAMPLE. If $D$ is a discrete space of measurable cardinal, then the relation $v(D \times \beta D)=v D \times \beta D$ is false. 
A cardinal number $\mathfrak{n}$ is said to be measurable if the discrete space of cardinality $\mathfrak{n}$ supports a countably additive measure assuming the values 0 and 1 (and only these values) and assigning measure 0 to each point. As is shown in Chapter 12 of [12], an equivalent condition is that the discrete space of cardinality $\mathfrak{n}$ is realcompact. The class of nonmeasurable cardinals being closed under the standard operations of cardinal arithmetic, it is consistent with the usual axioms of set theory to assume that each cardinal number is nonmeasurable. The consistency of the existence of measurable cardinals with the usual axioms of set theory remains an unsettled question.

2. Conditions sufficient for the relation $v(X \times Y)=v X \times v Y$. Our first result is a direct corollary to Theorem 1.2 quoted above.

2.1 THEOREM. Let $Y$ be a locally compact space of nonmeasurable cardinal. Then $X \times Y$ is $C^{*}$-embedded in $v X \times Y$, for each space $X$.

Proof. For each $f$ in $C^{*}(X \times Y)$ and each point $y$ in $Y$ there is a continuous real-valued function $g_{y}$ on $v X \times\{y\}$ which agrees with $f$ on $X \times\{y\}$. We define $g$ on $v X \times Y$ by the relation

$$
g=\bigcup_{y \in Y} g_{y}
$$

For $K \subset Y$ the function $g$ has a restriction to $v X \times K$ which is, according to Theorem 1.2 , continuous whenever $K$ is compact. To check the continuity of $g$ at an arbitrary point $(p, y)$ of $v X \times Y$, then, we need only find a compact neighborhood $K$ of $y$ and notice that $g$ is continuous on $v X \times K$.

2.2 COROLlARY. If $Y$ is a locally compact, realcompact space of nonmeasurable cardinal, then $v(X \times Y)=v X \times Y$ for each space $X$.

Proof. From 1.1 and 2.1.

The rôle played by compact subsets of $Y$ in the proof of 2.1 suggests the pertinence, to the problem under consideration, of those spaces whose topology is determined by compact subsets. The property may be formalized in two ways.

Definition. A space is said to be a $k$-space if each of its subsets which has closed intersection with each compact subset is itself closed.

Definition. A space is said to be a $k^{\prime}$-space if each real-valued function which has continuous restriction to each compact subset is continuous, i.e., if the space of continuous real-valued functions is complete in the structure of uniform convergence on compacta.

Evidently each $k$-space is a $k^{\prime}$-space, but Pták presents in [21] an example (credited to Katětov) which shows that the converse implication can fail. Another (completely regular Hausdorff) example, to which we will refer in $\$ 5$ below, has been discovered by Norman Noble in [20].

An extremely brief treatment of $k$-spaces occurs in [19], where it is shown on p. 231 that locally compact spaces, and first-countable spaces, are $k$-spaces. A 
more rewarding account of $k$-spaces and $k^{\prime}$-spaces appears in Seth Warner's very readable paper [23].

An apology is perhaps in order for the distasteful, artificial hypothesis which occurs in many of the results to be presented next: " $v X$ is locally compact." Easy examples show that the hypothesis cannot be omitted, but the question naturally arises whether this hypothesis can be replaced by a condition on $X$ itself. We devote $\S 4$ to this question.

2.3 THEOREM. If $Y$ is a $k$-space each of whose compact subsets is of nonmeasurable cardinal, and if $v X$ is locally compact, then $X \times Y$ is $C^{*}$-embedded in $v X \times Y$.

Proof. Given $f$ in $C^{*}(X \times Y)$, we define

$$
g=\bigcup_{y \in Y} g_{y},
$$

where just as in the proof of $2.1 g_{y}$ is that unique continuous function on $v X \times\{y\}$ which agrees with $f$ on $X \times\{y\}$. We know from Theorem XII.4.3 of [9] that a product of any $k$-space with a locally compact space is a $k$-space. Thus, in order to show that $g$ is continuous on $v X \times Y$, we need only show that the restriction of $g$ to each compact subset $K$ of $v X \times Y$ is continuous on $K$. Denoting by $\pi$ the projection from $v X \times Y$ onto $Y$, we see from Theorem 1.2 (applied to $K$ in place of $Y$ ) that $g$ is continuous on the set $v X \times\left(\pi^{-1}(\pi K)\right)$.

We will see in 4.5 that the cardinality restriction in our next theorem can be weakened.

2.4 THEOREM. Let $X \times Y$ be of nonmeasurable cardinal, where $Y$ is a $k$-space and $v X$ is locally compact. Then $v(X \times Y)=v X \times v Y$.

Proof. Theorem 2.3 shows that $X \times Y$ is $C^{*}$-embedded in $v X \times Y$. Theorem 2.1 shows that $v X \times Y$ is $C^{*}$-embedded in $v X \times v Y$. (The rôle of $X$ in Theorem 2.1 is played here by $Y$; and of $Y$, by $v X$. The theorem is applicable because, as 9.2 of [12] shows, $v X$ is of nonmeasurable cardinal whenever $X$ is.)

2.5 COROLlary. Let $X \times Y$ be of nonmeasurable cardinal, where $Y$ is a $k$-space and $X$ is pseudocompact. Then $v(X \times Y)=v X \times v Y$.

Proof. The space $X$, being pseudocompact, is $C$-embedded in $\beta X$. Thus $v X$ is the compact space $\beta X$.

The following result, a corollary to 2.5 , is a weakened version of Tamano's Proposition 2 in [22]. The present weakness rests in the fact that, as Tamano's statement shows, the cardinality restriction imposed here is inessential and may be omitted. Doubtless the general Tamano theorem can be deduced from the version given here, but I have not found the argument.

It seems only fair to remark also that Tamano's theorem is subsumed, at least formally, by Frolík's Theorem 3.6 in [11]. That theorem appears difficult to apply in practice, but its aesthetically displeasing content is more than compensated by 
its form: It lists, for a fixed space $X$, a necessary and sufficient condition that $X \times Y$ be pseudocompact for each pseudocompact space $Y$.

2.6 Corollary. Let $X$ and $Y$ be pseudocompact spaces of nonmeasurable cardinal, and let $X$ be a $k$-space. Then $X \times Y$ is pseudocompact.

Proof. Any space whose Hewitt realcompactification is compact is surely pseudocompact. The space $X \times Y$ is such a space, since from 2.5 follows the relation

$$
v(X \times Y)=v X \times v Y=\beta X \times \beta Y .
$$

The next theorem is a variation on 2.4 .

2.7 TheOREM. Let $X \times Y$ be of nonmeasurable cardinal, and let both $v X \times Y$ and $v X \times v Y$ be $k$-spaces. Then $v(X \times Y)=v X \times v Y$.

Proof. For each $f$ in $C^{*}(X \times Y)$ there is, just as before, a $g$ in $C^{*}(v X \times Y)$ which agrees with $f$ on $X \times Y$. Now for each $p \in v X$ let $h_{p}$ be a continuous real-valued function on $\{p\} \times v Y$ which agrees with $g$ on $\{p\} \times Y$. Since $v X \times v Y$ is a $k$-space we have, as before, that the function $h$, defined on $v X \times v Y$ by the relation

$$
h=\bigcup_{p \in \cup Y} h_{p},
$$

lies in $C^{*}(v X \times v Y)$. Theorem 1.1 completes the proof.

2.8 REMARK. The discussion which follows is designed to show that the hypothesized condition " $v X \times Y$ is a $k$-space" of 2.7 does not follow from the hypothesis " $v X \times v Y$ is a $k$-space."

It follows from Theorem 3.1 of [10], or from Lemma 2 of [4], that if $A$ is a dense subset of $\beta N \backslash N$, where $N$ denotes the countably infinite discrete set, then $N \cup A$ is pseudocompact. (The paper [4] discusses explicitly only the case

$$
\begin{aligned}
& A=\{\sigma(p): p \text { is a fixed point in } \beta N \backslash N \text {, and } \sigma \text { is the restriction } \\
& \text { to } \beta N \backslash N \text { of a homeomorphism of } \beta N \text { onto } \beta N\} .)
\end{aligned}
$$

Now let $A$ be a dense subset of $\beta N \backslash N$ for which $|A| \leqq c$, as afforded by Lemma 1 of [4] or by Theorem 4.1 of [3]. Since (from Corollary 9.12 of [12]) every infinite compact subset of $\beta N \backslash N$ has cardinality $2^{\mathfrak{c}}$, each compact subset of $N \cup A$ is finite. Thus every real-valued function on $N \cup A$ has a continuous restriction to each compact set, so that $N \cup A$ is not a $k^{\prime}$-space.

The paragraph above shows that there exists a pseudocompact space $Y=N \cup A$ which is not a $k$-space. For any such $Y$ and any space $X$ for which $v X$ is a $k$-space, we know that $v X \times v Y$ is a $k$-space; while $v X \times Y$ is not.

3. Statement of A. W. Hager's theorem. The Glicksberg-Frolík theorem quoted earlier can be viewed as a theorem which gives a condition sufficient for the relation $v(X \times Y)=v X \times v Y$ to hold. For it is easy to see (and is shown in 8A.4 of 
[12]) that a space $X$ is pseudocompact if and only if $v X=\beta X$. Thus one of the two Glicksberg-Frolík implications yields: If $X \times Y$ is pseudocompact, then

$$
v(X \times Y)=\beta(X \times Y)=\beta X \times \beta Y=v X \times v Y .
$$

In the interest of completeness we state here another theorem in the same vein, due to A. W. Hager. The expression "completely separated" in the next definition goes back at least to [2]. The suggestive symbol $\perp$ was introduced in this connection by F. W. Anderson in [1].

Definition. Two subsets $A$ and $B$ of the space $X$ are said to be completely separated (in $X$ ) if there exists $f \in C^{*}(X)$ such that $f \equiv 0$ on $A$ and $f \equiv 1$ on $B$. If $A$ and $B$ are completely separated in $X$, we write $A \perp_{X} B$ or, when confusion is impossible, simply $A \perp B$.

The following result, whose proof rests on the Glicksberg-Frolík theorem, is Theorem 1 of 3.6 of [15].

THEOREM. Let $X=\bigcup_{n=1}^{\infty} X_{n}$ and $Y=\bigcup_{n=1}^{\infty} Y_{n}$, and suppose that for each $n$ the space $X_{n} \times Y_{n}$ is pseudocompact and that $X_{n} \perp_{X}\left(X \backslash X_{n+1}\right)$ and $Y_{n} \perp_{Y}\left(Y \backslash Y_{n+1}\right)$. Then $v(X \times Y)=v X \times v Y$.

4. On the local compactness of $v X$. The next theorem is basic to all that follows. The proof offered here, because it is direct, seems preferable to the proof credited to D. G. Johnson by Hager in [15].

4.1 TheOREM (HAGER-Johnson). Let $U$ be an open subset of $X$, and suppose that $\mathrm{cl}_{\mathrm{DX}} U$ is compact. Then $\mathrm{cl}_{X} U$ is pseudocompact.

Proof. Suppose, on the contrary, that there is an unbounded real-valued continuous function $f$ with domain $\operatorname{cl}_{x} U$. Beginning with any point $x_{1}$ in $U$, we construct inductively a sequence of points $x_{n}$ in $U$ for which $\left|f\left(x_{n+1}\right)\right|>\left|f\left(x_{n}\right)\right|+1$. There is for each $n$ an element $g_{n}$ of $C(X)$ for which $g_{n}\left(x_{n}\right)=n$ and $g_{n}(x)=0$ whenever $\left|f(x)-f\left(x_{n}\right)\right|<1 / 4$, and it is easy to check that the function $g$ defined on $X$ by the relation $g=\sum_{n=1}^{\infty} g_{n}$ is continuous on $X$. The continuous extension of $g$ to $v X$ is, like $g$ itself, unbounded on $\operatorname{cl}_{X} U$, contradicting the hypothesis that this set is compact.

The concept "locally pseudocompact" has not, so far as I am aware, been introduced explicitly into the literature. Two possible definitions seem natural, and an easy proposition (noted in passing by Frolík in [11]) shows that for completely regular Hausdorff spaces they coincide.

4.2 Proposition. Let $V$ be an open subset of the pseudocompact space $U$. Then $\mathrm{cl}_{U} V$ is pseudocompact.

Proof. If $\left\{V_{n}\right\}_{n=1}^{\infty}$ is a sequence of relatively open subsets of $\mathrm{cl}_{U} V$ which is locally finite in $\operatorname{cl}_{U} V$, then $\left\{V_{n} \cap V\right\}_{n=1}^{\infty}$ is locally finite in $U$.

4.3 Definition. A space $X$ is locally pseudocompact at the point $x$ if either of the following equivalent conditions is satisfied: 
(a) $x$ admits a pseudocompact neighborhood;

(b) there is a local basis at $x$ of pseudocompact neighborhoods.

The following theorem will serve, in effect, as a lemma for part of the proof of 4.8 ; but it seems worthy of individual mention. The failure of its converse is given by an example referred to in $\S 5$.

4.4 THeOREM. Let $v X$ be locally compact. Then $X$ is locally pseudocompact.

Proof. For each $x$ in $X$ there is an open subset $W$ of $v X$ for which $x \in W$ and $\operatorname{cl}_{v X} W$ is compact. With $U=W \cap X$ we have $\operatorname{cl}_{v X} U=\mathrm{cl}_{\nu X} W$, so that $\operatorname{cl}_{X} U$ is by Theorem 4.1 a pseudocompact neighborhood of $x$ in $X$.

We can now give the promised generalization of Theorem 2.4.

4.5 THEOREM. Let each compact subset of $Y$, and each pseudocompact subset of $v X$, be of nonmeasurable cardinal, where $Y$ is a $k$-space and $v X$ is locally compact. Then $v(X \times Y)=v X \times v Y$.

Proof. The desired result will follow from Theorems 2.3, 2.1 and 1.1, once we have established that each compact subset $K$ of $v X$ has nonmeasurable cardinal. There is an open subset $U$ of $v X$ for which $K \subset U \subset \mathrm{cl}{ }_{\mathrm{D} X} U$, the last set being compact. We set

$$
A=\mathrm{cl}_{X}(U \cap X),
$$

so that $A$ is a pseudocompact subset of $X$ by 4.1. Since $v X$ is a Hausdorff space we have (from the relation $K \subset \mathrm{cl}_{v X} A$ ) that

$$
|K| \leqq\left|\mathrm{cl}_{\nu X} A\right| \leqq 2^{2|A|},
$$

so that $K$, like $A$, is of nonmeasurable cardinal.

A. W. Hager has shown in [15] that the space $v X$ is simultaneously locally compact and $\sigma$-compact if and only if $X$ has the form $X=\bigcup_{n=1}^{\infty} X_{n}$, where each $X_{n}$ is pseudocompact and $X_{n} \perp\left(X \backslash X_{n+1}\right)$ for each positive integer $n$. The ideas used in the next proof are very similar to his. This theorem is presented not because the condition described is elegant or easy to verify (it isn't), but because it is so far as I am aware the only condition known to be equivalent to the local compactness of $v X$.

4.6. THEOREM. In order that $v X$ be locally compact, it is necessary and sufficient that for each $p \in v X$ there exist pseudocompact subsets $A$ and $B$ of $X$ for which $p \in \mathrm{cl}_{\mathrm{vX}} A$ and $A \perp_{X}(X \backslash B)$.

Proof. Necessity. Given a compact neighborhood $K$ in $v X$ of $p \in v X$, let $f$ be a continuous function mapping $v X$ into $[0,1]$ with $f p=0$ and $f \equiv 1$ on $v X \backslash K$, and set

$$
A=\operatorname{cl}_{X}\left(X \cap f ^ { - 1 } \left[0,1 / 3[), B=\operatorname{cl}_{X}\left(X \cap f^{-1}[0,2 / 3[) .\right.\right.\right.
$$

An appeal to 4.1 completes the proof.

Sufficiency. To find a compact neighborhood of the point $p \in v X$, let $A$ and $B$ 
be as hypothesized and find a nonnegative function $f$ in $C^{*}(X)$ for which $f \equiv 0$ on $A$ and $f \equiv 1$ on $X \backslash B$. Let $g$ denote the continuous extension of $f$ to $\beta X$ and set $K=g^{-1}[0,1 / 2]$. Then $K$ is a compact neighborhood of $p$ in $\beta X$, so we need only show that $K \subset v X$. Like every pseudocompact subset of $X, B$ has compact closure in $v X$ (see 8.10 and $5 H .2$ of [12]). Thus, to show $K \subset v X$, we need only show $K \subset \mathrm{cl}_{\beta X} B$. But this is obvious, since if $q \in \beta X$ and $q \notin \mathrm{cl}_{\beta X} B$, then $q \in \mathrm{cl}_{\beta X}(X \backslash B)$, so that $g q=1$.

G. G. Gould shows in [14] how to associate with each suitably restricted family of subsets of $X$ some compactification of $X$. His theorems are not relevant to this paper, but the conditions he imposes seem well suited to our purposes.

4.7 Definition. A family $\mathscr{B}$ of subsets of $X$ is said to be a Gould bounding system provided

(i) $\bigcup \mathscr{B}=X$;

(ii) if $A_{1} \in \mathscr{B}$ and $A_{2} \in \mathscr{B}$, then $A_{1} \cup A_{2} \subset B$ for some $B \in \mathscr{B}$;

(iii) for each $A \in \mathscr{B}$ there exists $B \in \mathscr{B}$ such that $A \perp(X \backslash B)$.

4.8 THEOREM. Consider the following properties which a (completely regular Hausdorff) space $X$ may possess.

(1) $v X$ is locally compact;

(2) the family of pseudocompact subsets of $X$ is a Gould bounding system;

(3) some family of pseudocompact subsets of $X$ is a Gould bounding system;

(4) there is a locally compact space $Y$ for which $X \subset Y \subset v X$;

(5) $X$ is locally pseudocompact.

These properties are related as follows:

$$
\text { (1) } \Rightarrow(2) \Rightarrow(3) \Leftrightarrow(4) \Leftrightarrow(5) \text {. }
$$

Proof. (1) $\Rightarrow(2)$. The relation $X=\bigcup_{x \in X}\{x\}$ makes condition (i) of 4.7 obvious, and (ii) is clear because the union of two pseudocompact sets is pseudocompact. For (iii) of 4.7 we recall (again from 8.10 and $5 \mathrm{H} .2$ of [12]) that if $A$ is a pseudocompact subset of $X$ then $\mathrm{cl}_{v X} A$ is compact. Thus there is a compact neighborhood $K$ in $v X$ of $\operatorname{cl}_{v X} A$ and a continuous function $f$ mapping $v X$ into [0,1] for which $f \equiv 0$ on $A$ and $f \equiv 1$ on $v X \backslash K$. The desired set $B$ is defined by the relation

$$
B=\operatorname{cl}_{X}\left(X \cap f^{-1}[0,1 / 2[) \text {. }\right.
$$

(2) $\Rightarrow(3)$. This is obvious.

$(3) \Rightarrow(4)$. If $\mathscr{Q}$ is the hypothesized family of pseudocompact subsets of $X$, we set

$$
Y=\bigcup_{Q \in \mathcal{Q}} \mathrm{cl}_{\mathrm{vX}} Q .
$$

The local compactness of $Y$ follows just as in the "Sufficiency" argument of Theorem 4.6 above.

$(4) \Rightarrow(5)$. The proof of Theorem 4.4 carries over verbatim, except that the first occurrence of the symbol " $v X$ " must be replaced by the symbol " $Y$ ". 
(5) $\Rightarrow(3)$. Let

$$
\mathscr{Q}=\left\{A \subset X: A \text { is pseudocompact, and } A \perp_{X}(X \backslash B)\right.
$$

for some pseudocompact subset $B$ of $X$ \}.

We must show that $\mathscr{Q}$ is a Gould bounding system.

Each point of $X$ admits a pseudocompact neighborhood, and therefore $\{x\} \in \mathscr{Q}$ whenever $x \in X$. Thus (i) of 4.7 is satisfied.

For (ii), suppose that $A_{1} \in \mathscr{Q}$ and $A_{2} \in \mathscr{Q}$, and for $1 \leqq k \leqq 2$ let $f_{k}$ be a continuous function from $X$ into $[0,1]$ such that $f_{k} \equiv 0$ on $A_{k}$ and $f_{k} \equiv 1$ on $X \backslash B_{k}$ for some pseudocompact subset $B_{k}$ of $X$. Defining $f=f_{1} \cdot f_{2}$, we see that $f \equiv 0$ on $A_{1} \cup A_{2}$ and $f \equiv 1$ on $X \backslash\left(B_{1} \cup B_{2}\right)$. Thus

$$
A_{1} \cup A_{2} \perp_{X}\left(X \backslash\left(B_{1} \cup B_{2}\right)\right),
$$

and (ii) is verified.

It remains only to show that if $A \in \mathscr{Q}$, then $A \perp_{X}(X \backslash B)$ for some pseudocompact set $B$ which belongs to 2 . We know that there exists a closed pseudocompact subset $C$ of $X$ and a continuous function $f$ on $X$ to $[0,1]$ for which $f \equiv 0$ on $A$ and $f \equiv 1$ on $X \backslash C$. Defining

$$
B=\operatorname{cl}_{X} f^{-1}[0,1 / 2[
$$

we see that $B \perp_{X}(X \backslash C)$ and that $B$, which is actually $\mathrm{cl}_{C} f^{-1}[0,1 / 2$ [, is pseudocompact (being the closure of an open subset of a pseudocompact space). Thus $A \subset B \in \mathscr{Q}$ and the proof is complete.

The question of the equivalence of the five conditions given in 4.8 will be settled in the negative in [5] by the example referred to in $\$ 5$ below.

It is observed in Theorem 2.2 of [6] that $v X$ is a Baire space if and only if $X$ is. The following result is an easy consequence.

4.9 Proposition. If $X$ satisfies any of the five conditions of Theorem 4.8, then $X$ is a Baire space (and hence is of second category).

Proof. We know that there is a locally compact space $Y$ for which $X \subset Y \subset v X$. Now $Y$ is Baire, and hence $v Y$ (which is $v X$ ), and hence $X$.

5. The referee's example of a locally compact space $X$ for which $v X$ is not a $k$-space. The presence in the preceding sections of hypotheses like " $v X$ is locally compact" and " $v X$ is a $k$-space" bring to mind the question of whether $v X$ is locally compact, or a $k$-space, whenever $X$ is. It is easy to show that if $D$ is a discrete space of measurable cardinal, then $D$ is a locally compact space whose completion $v D$ is not even a $k^{\prime}$-space. (According to $8 \mathrm{~A} .5$ of [12], $v D$ is a $P$-space, so that by $4 \mathrm{~K} .3$ and $4 \mathrm{~K} .4$ of [12] each of its compact subsets is finite. Thus every real-valued function on the nondiscrete space $v D$ is continuous on each compact subset of $v D$.) Because this example depends on the existence of measurable cardinals, it seems dishonest or, at best, artificial; the question has arisen in frequent 
conversations whether a "nonmeasurable" locally compact space $X$ exists whose realcompactification $v X$ is not a $k$-space. In the original version of this paper we posed this question and left it open, remarking only that there is described in detail in [5] a $\sigma$-pseudocompact locally compact space $X$ for which $v X$ is not locally compact; and that Norman Noble has shown in [20] that if $A$ is an uncountable index set and $N_{\alpha}$ the countably infinite discrete space for each $\alpha \in A$, then the Corson $\Sigma$-space (defined as in [8])

$$
\left\{x \in \prod_{\alpha \in A} N_{\alpha}: x_{\alpha}=0 \text { except for countably many } \alpha \in A\right\}
$$

is a $k$-space whose realcompactification is, according to Theorem 2 of [8], just the $k^{\prime}$-space $\prod_{\alpha \in A} N_{\alpha}$, which is easily seen (by the technique suggested in $7 \mathrm{~J}(\mathrm{~b})$ of [19], for example) not to be a $k$-space.

The example which follows, and the arguments accompanying it, are due to the referee. It is a pleasure to repeat my thanks for his generosity. His construction is subtle in the following sense: Although the constructed space $X$ is evidently locally compact, the proof that $v X$ is not a $k$-space is indirect, since in fact the space $v X$ is never identified in concrete form. We learn very little about that space, except that it is not a $k$-space.

Let $\omega_{2}$ denote the smallest ordinal number of cardinality $\aleph_{2}$, let $Y$ denote the compact product space $\left(\omega_{2}+1\right) \times\left(\omega_{2}+1\right)$, and define

$$
X=\{(\sigma, \tau) \in Y: \sigma<\tau\} .
$$

The closure in $Y$ of the locally compact space $X$ is a compactification of $X$, so there is a continuous function $f$ mapping $\beta X$ onto $\operatorname{cl}_{Y} X$. We will show that $v X$ is not a $k$-space by showing that its subset

$$
A=\left\{p \in v X: f(p)=(\sigma, \sigma) \text { for some } \sigma<\omega_{2}\right\}
$$

is not closed, although it meets each compact subset of $v X$ in a closed set. For this latter property let $K$ be compact in $v X$, let $p_{\alpha}$ be any net in $A \cap K$ converging to $p$, and let $f(p)=(\tau, \tau)$. If $(\tau, \tau) \neq f\left(p_{\alpha}\right)$ for each index $\alpha$, then $\tau$ may be expressed in the form $\tau=\sup _{n \in N} \sigma_{n}$, where each of the ordinal numbers $\sigma_{n}$ satisfies the relation $\sigma_{n}<\tau$. It is now easy to construct a continuous real-valued function on $X$ unbounded on each neighborhood of $(\tau, \tau)$, contradicting the fact that $(\tau, \tau)$ is the image under $f$ of a point in $v X$. It follows that $p \in f^{-1}\left(f\left(p_{\alpha}\right)\right) \subset A$ for some $\alpha$, so that $p \in A \cap K$ as was to be shown.

To see that $A$ is not closed in $v X$, notice first that the set $\left\{\left(\sigma, \omega_{2}\right): \sigma<\omega_{2}\right\}$, being a noncompact, pseudocompact subset of $X$, is not closed in $v X$. This yields a point $q \in v X \backslash X$ for which $f(q)=\left(\omega_{2}, \omega_{2}\right)$.

It is obvious that $q \notin A$. The rest of this proof is devoted to showing that $q \in \mathrm{cl}_{\mathrm{vX}} A$.

Just as in the selection of $q$, we notice that for each $\tau<\omega_{2}$, the set $\{(\sigma, \tau): \sigma<\tau\}$ is pseudocompact whenever $\tau$ is not a limit of countably many smaller ordinal 
numbers; so there is for each such $\tau$ a point $p_{\tau}$ in $A$ for which $f\left(p_{\tau}\right)=(\tau, \tau)$, and in fact

$$
p_{\tau} \in \operatorname{cl}_{\nu X}\{(\sigma, \tau): \sigma<\tau\} .
$$

Now suppose that indeed $q \notin \mathrm{cl}_{v X} A$. Then some $\varphi \in C(v X)$ has the properties

$$
\varphi(q)=0, \quad \varphi \equiv 1 \text { on } A,
$$

and the preceding sentences show that

$$
\varphi \equiv 0 \text { on }\left(\sigma_{0}, \omega_{2}\right) \times\left\{\omega_{2}\right\} \quad \text { for some } \sigma_{0}<\omega_{2}
$$

and that for each non-first-countable ordinal $\tau<\omega_{2}$ we have

$$
\varphi \equiv 1 \quad \text { on } \quad(g(\tau), \tau) \times\{\tau\} \quad \text { for some } g(\tau)<\tau .
$$

We claim that the function $g$ just defined has the property that for some ordinal number $\sigma_{1}<\omega_{2}$ the relation $g(\tau)<\sigma_{1}$ is valid for cofinally many non-first-countable $\tau$. If this is not the case then for each $\tau$ we have a (minimal) $h(\tau)>\tau$ for which $g(\sigma) \geqq \tau$ whenever $\sigma \geqq h(\tau)$. The function $h$ can be used to define inductively a subset $\left\{\tau_{\alpha}: \alpha<\omega_{1}\right\}$ of $\omega_{2}$ indexed by the first uncountable ordinal: one defines $\tau_{1}$ arbitrarily in $\omega_{2}, \tau_{\alpha}=h\left(\tau_{\alpha-1}\right)$ if $\alpha$ is not a limit ordinal, and $\tau_{\alpha}=\sup \left\{\tau_{\gamma}: \gamma<\alpha\right\}$ for limit ordinals $\alpha<\omega_{1}$. Defining $\bar{\tau}=\sup _{\alpha<\omega_{1}} \tau_{\alpha}$ we see that $\bar{\tau}$ is not first-countable and that $\bar{\tau}>\tau_{\alpha}$ for each $\alpha$. It follows that $g(\bar{\tau}) \geqq \bar{\tau}$, a contradiction proving the existence of the desired ordinal $\sigma_{1}$.

Now let $\omega_{2}>\sigma>\sigma_{0}$ and $\sigma>\sigma_{1}$ and let $T$ be a cofinal (in $\omega_{2}$ ) subset of non-firstcountable ordinals for which $g(\tau)<\sigma_{1}$ whenever $\tau \in T$. Then $\varphi(\sigma, \tau)=1$ whenever $\tau \in T$, so the continuity of $\varphi$ forces the relation $\varphi\left(\sigma, \omega_{2}\right)=1$, contrary to the condition $\varphi\left(\sigma, \omega_{2}\right)=0$. It follows, then, that $q \in \mathrm{cl}_{v X} A \backslash A$, so that $v X$ is not a $k$-space. The proof is complete.

The referee has posed for consideration the question of whether each locally compact space $X$ of cardinality $\aleph_{1}$ or less has the property that $v X$ is a $k$-space. In posing this question he is motivated not simply by the space above, whose cardinality is $\boldsymbol{\aleph}_{2}$, but also by a space studied by Leonard Gillman and Melvin Henriksen in their paper Concerning rings of continuous functions, Trans. Amer. Math. Soc. 77 (1954), 340-362. This latter space, also of cardinality $\aleph_{2}$, is known to be of minimal cardinality with respect to a certain natural property.

\section{REFERENCES}

1. Frank W. Anderson, Approximation in systems of real-valued continuous functions, Trans. Amer. Math. Soc. 103 (1962), 249-271.

2. Eduard Cech, On bicompact spaces, Ann. of Math. 38 (1937), 823-844.

3. W. W. Comfort, Retractions and other continuous maps from $\beta X$ onto $\beta X \backslash X$, Trans. Amer. Math. Soc. 114 (1965), 1-9.

4. - A nonpseudocompact product space whose finite subproducts are pseudocompact, Math. Ann. 170 (1967), 41-44. 
5. W.W. Comfort, Locally compact realcompactifications, General Topology and its Relations to Modern Analysis and Algebra II, Proceedings of the Second Prague Topological Symposium, 1966, pp. 95-100.

6. W. W. Comfort and Stelios Negrepontis, The ring $C(X)$ determines the category of $X$, Proc. Amer. Math. Soc. 16 (1965), 1041-1045.

7. - Extending continuous functions on $X \times Y$ to subsets of $\beta X \times \beta Y$, Fund. Math. 59 (1966), 1-12.

8. H. H. Corson, Normality in subsets of product spaces, Amer. J. Math. 81 (1959), 785-796.

9. James Dugundji, Topology, Allyn and Bacon, Boston, Mass., 1966.

10. N. J. Fine and L. Gillman, Remote points in $\beta R$, Proc. Amer. Math. Soc. 13 (1962), 29-36.

11. Ždeněk Frolik, The topological product of two pseudocompact spaces, Czechoslovak. Math. J. 10 (1960), 339-349.

12. Leonard Gillman and Meyer Jerison, Rings of continuous functions, Van Nostrand, Princeton, N. J., 1960.

13. Irving Glicksberg, Stone-Čech compactifications of products, Trans. Amer. Math. Soc. 90 (1959), 369-382.

14. G. G. Gould, A Stone-Čech-Alexandroff-type compactification and its application to measure theory, Proc. London Math. Soc. (3) 14 (1964), 221-244.

15. Anthony Wood Hager, On the tensor product of function rings, Doctoral dissertation, Pennsylvania State Univ., University Park, 1965.

16. Edwin Hewitt, Rings of real-valued continuous functions. I, Trans. Amer. Math. Soc. 64 (1948), 45-99.

17. John R. Isbell, Uniform spaces, Math. Surveys No. 12, Amer. Math. Soc., Providence, R. I., 1964.

18. N. Onuchic, On the Nachbin uniform structure, Proc. Amer. Math. Soc. 11 (1960), 177-179.

19. John L. Kelley, General topology, Van Nostrand, Princeton, N. J., 1955.

20. Norman Noble, Doctoral dissertation, Univ. of Rochester, Rochester, N. Y., 1967.

21. Vlastimil Pták, On complete topological linear spaces, Czechoslovak. Math. J. 78 (1953), 301-364.

22. Hisahiro Tamano, A note on the pseudocompactness of the product of two spaces, Mem. Coll. Sci. Univ. Kyoto 33 (1960), 225-230.

23. Seth Warner, The topology of compact convergence on continuous function spaces, Duke Math. J. 25 (1958), 265-282.

UNIVERSITY OF MASSACHUSETTS, AMHERST, MASSACHUSETtS

WESLEYAN UNIVERSITY, MidDletown, ConNecticut 